\title{
2 Research Square \\ Predictive factors of selective mineralocorticoid antagonist treatment in chronic central serous chorioretinopathy
}

\section{Róbert Gergely ( $\sim$ gergelyrobert.dr@gmail.com )}

Semmelweis University https://orcid.org/0000-0002-5486-4707

\section{Illés Kovács}

Semmelweis Egyetem

\section{Gábor László Sándor}

Semmelweis Egyetem

\section{Cecília Czakó}

Semmelweis Egyetem

\section{Zsuzsanna Récsán}

Semmelweis Egyetem

\section{Zoltán Zsolt Nagy}

Semmelweis Egyetem

\section{Mónika Ecsedy}

Semmelweis Egyetem

\section{Research article}

Keywords: chronic central serous chorioretinopathy, predictive factors, mineralocorticoid receptor antagonist therapy, eplerenone

Posted Date: September 8th, 2019

DOI: https://doi.org/10.21203/rs.2.14178/v1

License: (a) This work is licensed under a Creative Commons Attribution 4.0 International License. Read Full License

Version of Record: A version of this preprint was published at Scientific Reports on October 6th, 2020. See the published version at https://doi.org/10.1038/s41598-020-73959-4. 


\section{Abstract}

Purpose: To compare the macular morphology of good and poor responders to eplerenone treatment in chronic central serous chorioretinopathy (CSCR) patients.

Methods: 30 eyes of 29 patients with chronic CSCR were treated with $50 \mathrm{mg} /$ day oral eplerenone and followed up for one year. The integrity of outer retinal layers at baseline was assessed using optical coherence tomography. Patients who showed complete resolution of subretinal fluid at one year were assigned to the good responder group (Group 1), whilst those who showed moderate or no resolution were classified as poor responders (Group 2).

Results: In Group 2 hyperreflective foci in outer segment and outer nuclear layer were significantly more frequent, while ellipsoid zone interruption was significantly more frequent than in Group 1 ( $p=0.006$ and $p<0.001$ respectively). The multivariable regression analysis showed that intact ellipsoid zone at baseline is an independent predictor of good therapeutic response, with an odds ratio of 26.00 (95\% Cl: 3.69183.45; $p=0.001$ ).

Conclusion: The resolution of subretinal fluid after eplerenone treatment is associated with the integrity of outer retinal layers in CSCR patients. Baseline morphologic evaluation of these layers can be useful in predicting the response to mineralocorticoid antagonist therapy.

\section{Background}

In the last five years our understanding of the pathophysiology of CSCR has changed dramatically after discovering that aldosterone plays an important role in retinal homeostasis and that excessive occupancy of the mineralocorticoid receptor (MR) by glucocorticoids induces MR activation, causing dilatation and leakage of the choroid vessels. ${ }^{1,2,3,4,5,6}$ Zhao et al. demonstrated that aldosterone controls the hydration of healthy retina by up-regulating the aquaporin 4 (AQP4) water channel in the retinal Müller cells through MR. ${ }^{6}$ In another study Zhao et al. proved that aldosterone specifically affects the choroidal vascular bed, but not the retinal. ${ }^{7}$ They showed that MR activation up-regulates the KCa2.3 channel that leads to hyperpolarization of endothelial cells and the underlying smooth muscle cells, thus inducing choroidal vasodilatation. ${ }^{7}$ Based on these results, it was hypothesized that the MR-antagonist eplerenone could be a possible treatment in CSCR. ${ }^{7}$ Bousquet et al found significant choroidal thinning and visual improvement after one to three months of eplerenone treatment in 13 patients with chronic CSCR. ${ }^{8}$ These results have been validated by numerous other studies. ${ }^{9}, 10,11,12$ We also showed in our previous prospective clinical study that 3 month eplerenone therapy can reverse the choroidal vasodilatation and subsequently induce reabsorption of subretinal fluid. ${ }^{13}$

Despite these promising results, we noticed that there are some patients who tend to react only modestly to treatment. This study was intended to assess if there are any factors which can differentiate the CSCR patients who would benefit promptly from eplerenone treatment. 


\section{Methods}

\section{Study design and patient recruitment}

In this retrospective cohort study, we included patients with chronic CSCR, presented between June 2014 and September 2017 at the Department of Ophthalmology of Semmelweis University, Hungary, who were treated with $50 \mathrm{mg} /$ day of oral eplerenone and followed for at least one year. Inclusion and exclusion criteria are summarized in Table 1. The patients who showed complete resolution of subretinal fluid at one year were assigned to the good responder group (Group 1), whilst those who showed moderate or no resolution were categorized as the poor responders (Group 2). CSCR patients were treated with oral eplenerone (Inspra ${ }^{\circledR}$, Pfizer) $25 \mathrm{mg} /$ day for a week, followed by $50 \mathrm{mg} /$ day for at least 3 months. Follow up visits were performed at three-month intervals of $3,6,9$, and 12 months. If any subretinal fluid was observed during spectral-domain optical coherence tomography (SD-OCT) examination after the 3-month eplerenone treatment, the therapy was continued or restarted up to the presence of subretinal fluid. The potential risks and benefits were thoroughly discussed with all patients and written informed consent was obtained before treatment.

\section{Baseline and follow-up examinations}

Every patient included in the study had a physical examination and a routine blood test before treatment -with the former being repeated each month if the treatment was continued. We recorded the patient's medical history, serum potassium level, and kidney function (estimated glomerular filtration rate). Previous ophthalmological treatment for CSCR (anti-VEGF, photodynamic therapy) was noted, if there was any. At baseline and at every follow-up, best-corrected visual acuity (BCVA) measurement with ETDRS charts at 4 meters was performed on both eyes of each patient (Snellen acuity and ETDRS letter score were noted; $20 / 20=85$ ). We also noted the duration of the disease and whether it was recurrent or not. The diagnosis of CSCR was based on slit lamp examination and optical coherence tomography (OCT) findings (localized and limited serous detachments of the neurosensory retina on the posterior pole, without any other findings). When needed for differential diagnosis, and to exclude choroidal neovascularization, we also performed fluorescein angiography and/or indocyanine green angiography. Macular morphology was examined with Spectral-Domain Optical Coherence Tomography (RTvue OCT ver.6, 11.0.12, Optovue Inc., Fremont, USA). Horizontal raster pattern scans centered on the fovea were used for the examination. The following morphological changes were noted: subfoveal and extrafoveal pigment epithelium detachments (PED), double layer signs (DLS), elongation of the photoreceptors' outer segment, integrity of the external limiting membrane (ELM), integrity of the ellipsoid zone, hyperreflective foci in the outer segment (OS) and outer nuclear (ON) layer, and subretinal deposits in the subretinal fluid. A layer was defined intact if there was no interruption in that layer on any of the OCT scans. Single or multifocal appearance was noted. Retinal thickness was measured automatically from the internal limiting membrane to Bruch's membrane in all the 9 subfields of the macula. For data analysis we used the central subfield mean thickness of the Early Treatment Diabetic Retinopathy Study (ETDRS, the 
central $1.0 \mathrm{~mm}$ diameter of the macula). Data were recorded at baseline and then every 3 months for one year. Any adverse events that occurred were recorded at each visit.

\section{Outcome measures}

The primary objective was to point out morphological differences seen on SD-OCT between the patients who responded well to eplerenone treatment and those who did not and to identify predictive factors of good therapeutic response.

The secondary objective was to assess the relationship between general risk factors (hypertension, hyperlipidemia), disease duration, reoccurrence and appearance type (mono or multifocal) with regards to treatment response.

\section{Statistical analysis}

Statistical analysis was performed with Statistica software (version 13.2, Statsoft Inc., Tulsa, OK, USA). The independent samples student's t-test and chi-squared test were applied for group comparisons. The paired samples t-test was used for statistical comparisons of repeated measurements. The effect of predisposing factors on treatment response was assessed with multivariable regression analysis using binomial logit models to estimate odds of good response after controlling for the effect of other risk factors. Covariates which were evaluated to be potential confounders were included where the parameters showed significant difference in group comparisons. In all statistical analyses, a p-value of less than 0.001 was considered to be statistically significant.

\section{Results}

30 eyes of 29 patients were included in the study. In the whole cohort, central retinal thickness decreased significantly ( $p=0.0001)$, while final visual acuity did not show significant improvement at the end of the one-year follow-up period $(p=0.78)$. We found complete resolution of subretinal fluid in 15 eyes $(51.72 \%)$ of 14 patients (Group 1), and 15 eyes of 15 patients showed no or moderate resolution (Group 2).

We found that the central subfield mean thickness decreased in both groups, but the change was more significant in good responder patients (Group 1). Patients in Group 1 gained 5.0 letters at 12 months compared to baseline, while Group 2 showed a decrease in visual acuity of 5.9 letters on average. Changes in central subfield mean thickness (CSMT) and visual acuity are summarized in Table 2 and Figure 1. / A, B, C, D.

There was no significant difference in baseline central subfield macular thickness, baseline visual acuity, disease duration, reoccurrence, appearance type (mono or multifocal), treatment duration and the presence of general risk factors (hypertension, hyperlipidemia) between the two groups. 
Baseline characteristics, demographic data, patient history, disease duration and treatment duration in the two groups are summarized in Table 3.

\section{Differences in morphological characteristics}

Ellipsoid zone integrity and hyperreflective foci in the OS and ON layer (also known as intra and subretinal precipitates) were found to be significant factors in differentiating mineralocorticoid antagonist treatment response. In patients who showed complete resolution of subretinal fluid at the one-year follow-up, a continuous ellipsoid zone was observed significantly more frequently, whilst hyperreflective foci in the OS and ON layer was observed to be significantly rarer than that recorded in the poor responder group (Table 4., Figure 2.).

Finally, multivariable binomial logistic regression analysis was performed to determine significant predictors of a good anatomical response to eplerenone treatment. According to the best fit model $\left(r^{2}: 0.43 ; p:<0.001\right)$ an intact ellipsoid layer was determined to be an independent predictor of fluid resolution, with an odds ratio of 26.00 (95\% Cl:3.69-183.45; $\mathrm{p}=0.001)$.

\section{Safety Analysis}

There were no serious events that occurred as a result of eplerenone treatment. Mild adverse events were seen in $20.68 \%$ of the cases (dry mouth, dizziness, back pain, and sleepiness for a few hours after taking the pills). The treatment with eplerenone was generally well tolerated and did not lead to elevated potassium levels or low creatinine clearance.

\section{Discussion}

In the last five years many papers showed that eplerenone is a safe and effective treatment option in chronic CSCR patients, mainly through reversing the choroidal vasodilatation that results in resolution of subretinal fluid. ${ }^{1,7-13}$

In this study, we have chosen fluid resolution as the biomarker of good response instead of the change in visual acuity, because neuroretinal re-attachement is not always accompanied by visual acuity improvement. Besides the presence of subretinal fluid several other factors are influencing the visual prognosis, such as the disruption of ellipsoid zone, disruption of the ELM, thinning of the ON layer, hyperreflective foci in the retinal layers and the severity of RPE atrophy. 1,15

Recent studies reported different predictors of treatment response in this new therapy. In our previous prospective study we found that in eplerenone treated CSCR patients baseline choroidal thickness was a positive predictive factor for subretinal fluid decrease, results which were recently supported by Bousquet et al. who found that a thick choroid at baseline is associated with treatment response. ${ }^{13,14}$ As for 
neuroretinal morphological changes Cakir and coworkers reported that CSCR patients with intact RPE and intact ellipsoid zone had better BCVA after eplerenone treatment. ${ }^{15}$ Sacconi et al. reported possible biomarkers on ICG-angiography and OCT-angiography which could predict the response to eplerenone treatment in CSCR patients. ${ }^{16}$ They found that the absence ofchoroidal neovascularization (CNV) on OCTangiography and the presence of hot spot on ICG-angiography were associated with a good response to treatment, with $58 \%$ of the patients (17 out of 29 ) showing a complete resolution of the subretinal fluid at the end of the 13 week treatment. ${ }^{16}$ Interestingly patients with CNV and hotspot also showed a moderate response to eplerenone therapy. ${ }^{16}$

In our study population ellipsoid zone integrity at baseline was found to be a crucial and independent predictor of good anatomical treatment response as defined by complete and stable resolution of subretinal fluid. At the same time, hyperreflective foci in the OS and ON layer showed a significant negative correlation with subretinal fluid resolution.

Within the outer retina, a hyperreflective band-previously thought to represent the photoreceptor inner/outer segment (IS/OS) junction, more commonly referred to now as the inner segment ellipsoidcan be observed on SD-OCT. The integrity of this layer has been found to be of high clinical importance in the diagnosis and prognosis evaluation of various surgical and medical retinal diseases. The absence or disruption of this layer has been found to be associated with lower visual acuity and poor visual prognosis. An intact ellipsoid layer was identified as a significant biomarker for macular hole, epiretinal membrane, and retinal detachment surgery, as well as eyes with wet age-related macular degeneration, retinal vein occlusion or diabetic macular edema. ${ }^{17}$ Damage of ellipsoid zone integritywas described in the natural course of CSCR, which also correlates with the macular function evaluated by microperimetry. ${ }^{18,19}$ In several SD-OCT studies, the ellipsoid zone was not visible in eyes with acute serous detachment, though it did became visible after its resolution. ${ }^{18,20,21}$ Koo et al. suggested that the membranous stack of the photoreceptor segment is no longer perpendicular to the incoming OCT beam in acute neurosensory retinal detachment, as the highly back-reflecting signal at the IS/OS seems to be absent in the area of detachment. ${ }^{22}$ Accordingly, even if an eye with active CSCR does not show a distinct ellipsoid zone, good visual acuity is often obtained when the subretinal fluid is resorbed. ${ }^{22,23}$ In contrast, in our study baseline ellipsoid layer damage was significantly associated with poor treatment response for mineralocorticoid antagonist therapy. We think that the reason for this incongruity is that only eyes with a chronic form of the disease were included in this study. In these cases,-as the IS/OS line is a hallmark of integrity of outer photoreceptor layer-ellipsoid integrity can show us that photoreceptors are preserved despite their long-lasting separation from retinal pigment epithelium and choroidal layers. In an experimental animal model of retinal detachment a significant decrease in the number ofphotoreceptors nuclei in the outer nuclear layer was observed at 1 month, followed by outer plexiform layer (OPL) degeneration within 50 days due to necrosis of cell processes and synaptic terminals. ${ }^{24}$ According to Piccolino et al.'s study, this is not the case in CSCR eyes where SD-OCT does not show atrophy of the OPL in detachments lasting several months, with a severe loss of the photoreceptor layer only being observed in patients with symptoms lasting more than one year. ${ }^{21}$ Our patients also had quite good baseline visual 
acuity despite the chronic presence of subretinal fluid, so for this reason the change in visual acuity will be smaller.

Hyperreflective foci in the OS and ON layer, as observed in all patients in the poor responder group, were the other significant biomarker predicting poor treatment response in our study population. These abnormal features of the OS and ON layer were earlier reported on the time-domain Stratus OCT images as a granulated profile of the outer border of neurosensory retina, mostly attenuated to the chronic or recurrent form of the disease..$^{21}$ Later in SD-OCT studies these hyperreflective foci in the OS and ON layer were identified in as many as $65 \%$ of chronic CSCR eyes in the area of serous retinal detachment ${ }^{20,25}$ and it co-localized with hyperautofluorescent areas on FAF images. ${ }^{26,27,28}$ The nature of these dots vary depending on their location. ${ }^{28}$ In the subretinal space these dots are thought to be macrophages and microglia activated by the photoreceptor outer segments shedding. ${ }^{27}$ Concentration of protein-like compounds, fibrin or lipids could also be identified as dots. ${ }^{25}$ Recently the observation of these hyperreflective foci in active and resolved CSCR eyes with adaptive optics scanning light ophthalmoscopy supported the hypothesis that they are cellular in nature, corresponding to activated microglia cells or macrophages. ${ }^{29}$ Their presence were found to be correlated not only to disease duration, but also to poorer final visual acuity. ${ }^{30,31}$

Interestingly, despite the fact that both biomarkers found in our study population were earlier attenuated to the chronic form of CSCR, no significant difference was found in disease duration or phenotype (recurrent or continuous, mono or multifocal) between the good and poor responder groups. One explanation for these results could be that in recurrent and chronic CSCR the duration of neurosensory detachment is often doubtful. The other important factor is that until now no consensus existed over the duration threshold that differentiates acute and chronic CSCR. In most published reports, it was arbitrarily set as being between 3 to 6 months and was used as a limit in interventional studies to determine the appropriate timing for treatment, in order to avoid self-resolution cases. As a result of which we consider that a consensual definition of the various clinical subtypes of CSCR is needed as their exact limits are critical in the determination of clinical trial design. We are of the opinion that morphologic assessment might better differentiate acute from chronic forms given that the duration of the disease seems to be an interval rather than an exact time following the appearance of the disease. These data suggests that the disease duration does not affect directly the response to eplerenone treatment. Previous study showed that the ON layer thickness positively correlates with the visual acuity in resolved CSCR and the discontinuity in the ellipsoid zone is associated with worse visual acuity. ${ }^{32} \mathrm{~A}$ recent study which reviewed clinical and multimodal imaging data of 133 patients with CSCR showed that outer retinal disruption on OCT is significantly associated with poorer visual acuity. ${ }^{33}$ These findings explains the incongruity of subretinal fluid absorbtion and visual acuity change in this disease, and shows that ellipsoid zone integrity is important in predicting the visual acuity. Nevertheless until now we had no data regarding if there is any correlation between the disruption of the ellipsoid zone and the anatomical response to eplerenone treatment in CSCR patients. 
Our study's limitations are the small sample size, the retrospective design and the absence of a placebotreated group. Nevertheless our cohort consisted of strictly selected cases and was followed-up for a relatively long period.

\section{Conclusions}

Our results suggest that in chronic or recurrent CSCR a morphologic evaluation of the macula may provide useful information not only about the present state of photoreceptor deterioration and potential for visual recovery, but also about the probability of subretinal fluid resolution. Next to choroidal thickness measurement the ellipsoid zone seems to be a useful biomarker for selecting the CSCR patients who could benefit the most from mineralocorticoid antagonist therapy.

\section{Abbreviations}

AQP4: aquaporin 4

BCVA: best-corrected visual acuity

CNV (choroidal neovascularisation)

CSCR: Central Serous Chorioretinopathy

CSMT: central subfield thickness

DLS: double layer signs

ELM: integrity of the external limiting membrane

MR: mineralocorticoid receptor

ON: outer nuclear

OPL: outer plexiform layer

OS: outer segment

PED: pigment epithelium detachments

SD-OCT: spectral-domain optical coherence tomography

\section{Declarations}

\section{Acknowledgment:}

None of the authors have a proprietary or financial interest. 


\section{Availability of data and materials:}

All the data supporting our findings is contained within the manuscript

\section{Author contributions:}

RG, ME-conception and design of the work, RG, GLS, CC, ME-acquisition of data, RG, KI, ME-analysis and interpretation of data, RG, IK, ZsR, ZZsN, ME-conceptualization of the manuscript and review and synthesis of the literature. All authors critically reviewed and revised the manuscript.

\section{Ethics approval and consent to participate:}

The study was conducted according to the Declaration of Helsinki, relevant national and local requirements, and was approved by the Ethical Review Board for Human Research of the National Drug Agency (ETT TUKEB, study number 104/2014, clinicaltrial.gov identifier NCT02462499).

\section{Consent for publication:}

Not applicable.

\section{Competing interests:}

The authors declare that they have no competing interests.

\section{References}

1. Daruich A, Matet A, Dirani A et al. Central serous chorioretinopathy: Recent findings and new physiopathology hypothesis. Prog Retin Eye Res 2015;48:82-118.

2. Fine HF, Ober MD, Hariprasad SM. Current concepts in managing central serous chorioretinopathy. Ophthalmic Surg Lasers Imaging Retina 2014;45:9-13.

3. Liew G, Quin G, Gillies M et al. Central serous chorioretinopathy: a review of epidemiology and pathophysiology. Clin Experiment Ophthalmol 2013;41:201-214.

4. Nicholson B, Noble J, Forooghian F et al. Central serous chorioretinopathy: update on pathophysiology and treatment. Surv Ophthalmol 2013;58:103-126.

5. Quin G, Liew G, Ho IV et al. Diagnosis and interventions for central serous chorioretinopathy: review and update. Clin Experiment Ophthalmol 2013;41:187-200.

6. Zhao M, Valamanesh F, Celerier I et al. The neuroretina is a novel mineralocorticoid target: aldosterone up-regulates ion and water channels in Muller glial cells. FASEB J 2010;24:3405-3415. 
7. Zhao M, Celerier I, Bousquet E et al. Mineralocorticoid receptor is involved in rat and human ocular chorioretinopathy. J Clin Invest 2012;122:2672-2679.

8. Bousquet E, Beydoun T, Zhao M et al. Mineralocorticoid receptor antagonism in the treatment of chronic central serous chorioretinopathy: a pilot study. Retina 2013;33:2096-2102.

9. Danjuma Ml, Mukherjee I, Makaronidis $\mathrm{J}$ et al. Converging indications of aldosterone antagonists (spironolactone and eplerenone): a narrative review of safety profiles. Curr Hypertens Rep 2014;16:414.

10. Herold TR, Prause K, Wolf A et al. Spironolactone in the treatment of central serous chorioretinopathy - a case series. Graefes Arch Clin Exp Ophthalmol. 2014;252:1985-1991.

11. Ghadiali Q, Jung JJ, Yu S et al. Central Serous Chorioretinopathy Treated with Mineralocorticoid Antagonists: A One-Year Pilot Study. 2016 Mar;36:611-618.

12. Pichi F, Carrai P, Ciardella A, Behar-Cohen F, Nucci P. Central Serous Chorioretinopathy Study Group. Comparison of two mineralocorticosteroid receptor antagonists for the treatment of central serous chorioretinopathy. Int Ophthalmol. 2016 Oct. 2017 Oct; 37(5):1115-11125.

13. Gergely R, Kovács I, Schneider M, Resch M, Papp A, Récsán Z, Nagy ZZ, Ecsedy M. Mineralocorticoid receptor antagonist treatment in bilateral chronic central serous chorioretinopathy: a comparative study of exudative and nonexudative fellow eyes. Retina. 2017 Jun;37:1084-1091.

14. Bousquet E, Dhundass M, Lejoyeux R, Shinojima A, KrisovicV, Mrejen S,Gaudric A, Tadayoni R. Predictive factors of response to mineralocorticoid therapy in nonresolving central serous chorioretinopathy. Am J Ophtalmol. 2019;198:80-87.

15. Cakir B, Fischer F, Ehlken C, Bühler A, Stahl A, Schlunck G et al. Clinical experience with eplerenone to treat chronic central serous chorioretinopathy. Graefes Arch Clin Exp Ophthalmol 2016;254:21512157.

16. Sacconi R, Baldwin G, Carnevali A, Querques L, Rabiolo A, Marchini G, Bandello F, Querques G. Response of central serous chorioretinopathy evaluated by multimodal retinal imaging. Eye 2018;32:734-742.

17. Saxena S, Srivastav K, Cheung CM, Ng YJ, Lai TY. Photoreceptor inner segment ellipsoid band integrity on spectral domain optical coherence tomography. Clinical Ophthalmology 2014;8:25072522.

18. Ojima A, lida T, Maruko I, Sugano Y. Photopigments in central serous chorioretinopathy. Am J ophthlamol. 2011;151:940-952.

19. Ozdemir H, Karacorlu SA, Senturk F, Karacorlu M, Uysal O. Assessment of macular function by microperimetry in unilateral resolved central serous chorioretinopathy. Eye (Lond) 2008;2:204-208.

20. Ojima Y, Hangai M, Sasahara M, Gotoh N, Inoue R, Yasuno Y, Makita S, Yatagai T, Tsujikawa A, Yoshimura N. Three-dimensional imaging of the foveal photoreceptor layer in central serous chorioretinopathy using high-speed optical coherence tomography. Ophthalmology. 2007 Dec;114:2197-2207. 
21. Piccolino FC, de la Longrais RR, Ravera G, Eandi CM, Ventre L, Abdollahi A, Manea M. The foveal photoreceptor layer and visual acuity loss in central serous chorioretinopathy. Am J Ophthalmol. 2005 Jan;139:87-99.

22. Ko TH, Fujimoto JG, Duker JS, et al. Comparison of ultrahigh-and standard-resolution optical coherence tomography for imaging macular hole pathology and repair. Ophthalmology 2004;111:2033-2043.

23. Ojima Y, Tsujikawa A, Yamashiro K et al. Restoration of outer segments of foveal photoreceptors after resolution of central serous chorioretinopathy. Jpn J Ophthalmol. 2010;54:55-60.

24. Erickson PA, Fisher SK Anderson DH Stern WA, Borgula GA. Retinal detachment in the cat: the outer nuclear and outer plexiform layers. Invest Ophthalmol Vis Sci. 1983 Jul;24:927-942.

25. Kon Y, lida T, Maruko I, Saito M. The optical coherence tomography-ophthalmoscope for examination of central serous chorioretinopathy with precipitates. Retina. 2008;28:864-869.

26. Iacono P, Battaglia PM, Papayannis A, La Spina C, Varano M, Bandello F. Acute central serous chorioretinopathy: a correlation study between fundus autofluorescence and spectral-domain OCT. Graefes Arch Clin Exp Ophthalmol. 2015 Nov; 253;11:1889-1897.

27. Maruko I, lida T, Ojima A, Sekiryu T. Subretinal dot-like precipitates and yellow material in central serous chorioretinopathy. Retina. 2011;31:759-765.

28. Spaide RF, Klancnik JM Jr, Cooney MJ. Retinal vascular layers imaged by fluorescein angiography and optical coherence tomography angiography. JAMA Ophthalmol. 2015;133:45-50.

29. Vogel RN, Langlo CS, Scoles D, Carroll J, Weinberg DV, Kim JE. High-Resolution Imaging of Intraretinal Structures in Active and Resolved Central Serous Chorioretinopathy. Invest Ophthalmol Vis Sci 2017;58:42-49.

30. Landa G, Barnett JA, Garcia PM, Tai KW, Rosen RB. Quantitative and qualitative spectral domain optical coherence tomography analysis of subretinal deposits in patients with acute central serous retinopathy. Ophthalmologica. 2013;230:62-68.

31. Yalcinbayir O, Gelisken O, Akova-Budak B, Ozkaya G, Gorkem Cevik S, Yucel AA. Correlation of spectral domain optical coherence tomography findings and visual acuity in central serous chorioretinopathy. Retina. 2014 Apr;34:705-712.

32. Matsumoto $H$, Sato T, Kishi S. Outer nuclear layer thickness at the fovea determines visual outcomes in resolved central serous chorioretinopathy. Am J Ophthalmol. 2009 Jul;148:105-10.

33. Mrejen S, Balaratnasingam C, Kaden TR, Bottini A, Dansingani K, Bhavsar KV, Yannuzzi NA, Patel S, Chen KC, Yu S, Stoffels G, Spaide RF, Freund KB, Yannuzzi LA. Long-term Visual Outcomes and Causes of Vision Loss in Chronic Central Serous Chorioretinopathy. Ophthalmology. 2019 Apr; $126(4): 576-588$.

\section{Tables}


Table 1. Patient Recruitment Criteria

Inclusion criteria

visual symptoms and clinical diagnosis of CSC for at least 3 months

presence of SRF involving the fovea on OCT and the SRF did not dissolve during the 3-

month period or did not decrease $>50 \mu \mathrm{m}$

\section{Exclusion criteria}

under 18 years of age or limited capacity

any evidence of choroidal neovascularization or choroidal hemangioma

history of other retinal abnormalities

liver or kidney disease

hyperkalemia $(>5.0 \mathrm{mmol} / \mathrm{L})$

high serum creatinine level $(>2 \mathrm{mg} / \mathrm{dL}$ in men, $>1.8 \mathrm{mg} / \mathrm{dL}$ in woman) or creatinine clearance $<50 \mathrm{~mL} /$ minute

concomitant therapy with drugs that increases the potassium level or are known to interact with eplerenone

pregnancy or planning to conceive

Table 2. Visual acuity and central subfield thickness (CSMT) during the follow-up.

\begin{tabular}{|c|c|c|c|c|}
\hline & Baseline & Month 3 & Month 6 & Month 12 \\
\hline \multicolumn{5}{|l|}{ Whole cohort } \\
\hline $\begin{array}{l}\text { Visual acuity (ETDRS } \\
\text { letter) }\end{array}$ & $72.63 \pm 12.22$ & $\begin{array}{c}75.03 \pm \\
12.83\end{array}$ & $\begin{array}{c}75.53 \pm \\
13.05\end{array}$ & $72.10 \pm 16.44$ \\
\hline CSMT $(\mu \mathrm{m})$ & $\begin{array}{c}359.13 \pm \\
89.24\end{array}$ & $\begin{array}{c}303.50 \pm \\
63.96\end{array}$ & $\begin{array}{c}274.37 \pm \\
47.67\end{array}$ & $\begin{array}{c}268.50 \pm \\
64.57^{*}\end{array}$ \\
\hline \multicolumn{5}{|l|}{ Good responders } \\
\hline $\begin{array}{l}\text { Visual acuity (ETDRS } \\
\text { letter) }\end{array}$ & $76.00 \pm 14.04$ & $\begin{array}{c}78.93 \pm \\
13.39\end{array}$ & $\begin{array}{c}79.73 \pm \\
13.62\end{array}$ & $81.00 \pm 10.72^{*}$ \\
\hline CSMT $(\mu \mathrm{m})$ & $\begin{array}{c}362.60 \pm \\
71.60\end{array}$ & $\begin{array}{c}302.00 \pm \\
60.85\end{array}$ & $\begin{array}{c}267.33 \pm \\
39.11\end{array}$ & $\begin{array}{c}238.67 \pm \\
44.44^{*}\end{array}$ \\
\hline \multicolumn{5}{|l|}{ Poor responders } \\
\hline $\begin{array}{l}\text { Visual acuity (ETDRS } \\
\text { letter) }\end{array}$ & $69.27 \pm 9.39$ & $\begin{array}{l}71.13 \pm \\
11.36\end{array}$ & $\begin{array}{c}71.33 \pm \\
11.37\end{array}$ & $63.20 \pm 16.58^{\dagger}$ \\
\hline CSMT $(\mu \mathrm{m})$ & $\begin{array}{c}355.67 \pm \\
106.51\end{array}$ & $\begin{array}{c}305.00 \pm \\
69.06\end{array}$ & $\begin{array}{c}281.40 \pm \\
55.41\end{array}$ & $\begin{array}{c}298.33 \pm \\
68.87^{\dagger}\end{array}$ \\
\hline
\end{tabular}


Note: *: $\mathrm{p}<0.01$ compared to baseline using repeated measures analysis of variance test †: $\mathrm{p}<0.01$ compared to good responders using the independent samples student's t-test.

Table 3. Baseline, disease, and treatment characteristics of the two groups.

Note: Independent samples student's t-test and chi-squared test were applied for group comparisons.

Table 4. Baseline OCT characteristics of the good (Group 1) and poor (Group 2) responders. 


\begin{tabular}{|c|c|c|c|}
\hline & $\begin{array}{l}\text { Group } 1 \\
(\mathrm{n}=15, \\
\text { good } \\
\text { responders) }\end{array}$ & $\begin{array}{l}\text { Group } 2 \\
(\mathrm{n}=15, \\
\text { poor } \\
\text { responders })\end{array}$ & $\mathrm{P}$ \\
\hline Double layer sign & $10 / 5$ & $12 / 3$ & 0.41 \\
\hline PED & $5 / 10$ & $4 / 11$ & 0.69 \\
\hline OS elongation & $13 / 2$ & $8 / 7$ & 0.046 \\
\hline $\begin{array}{l}\text { Hyperreflective foci in } \mathrm{OS} \text { and } \mathrm{ON} \\
\text { layer }\end{array}$ & $9 / 6$ & $15 / 0$ & $0.006^{*}$ \\
\hline Subretinal deposits & $2 / 13$ & $2 / 13$ & 0.99 \\
\hline Ellipsoid integrity & $12 / 3$ & $2 / 13$ & $0.0003^{*}$ \\
\hline ELM integrity & $12 / 3$ & $6 / 9$ & 0.02 \\
\hline
\end{tabular}

Note: *: $\mathrm{p}<0.01$ compared to good responders using chi-squared test.

\section{Figures}



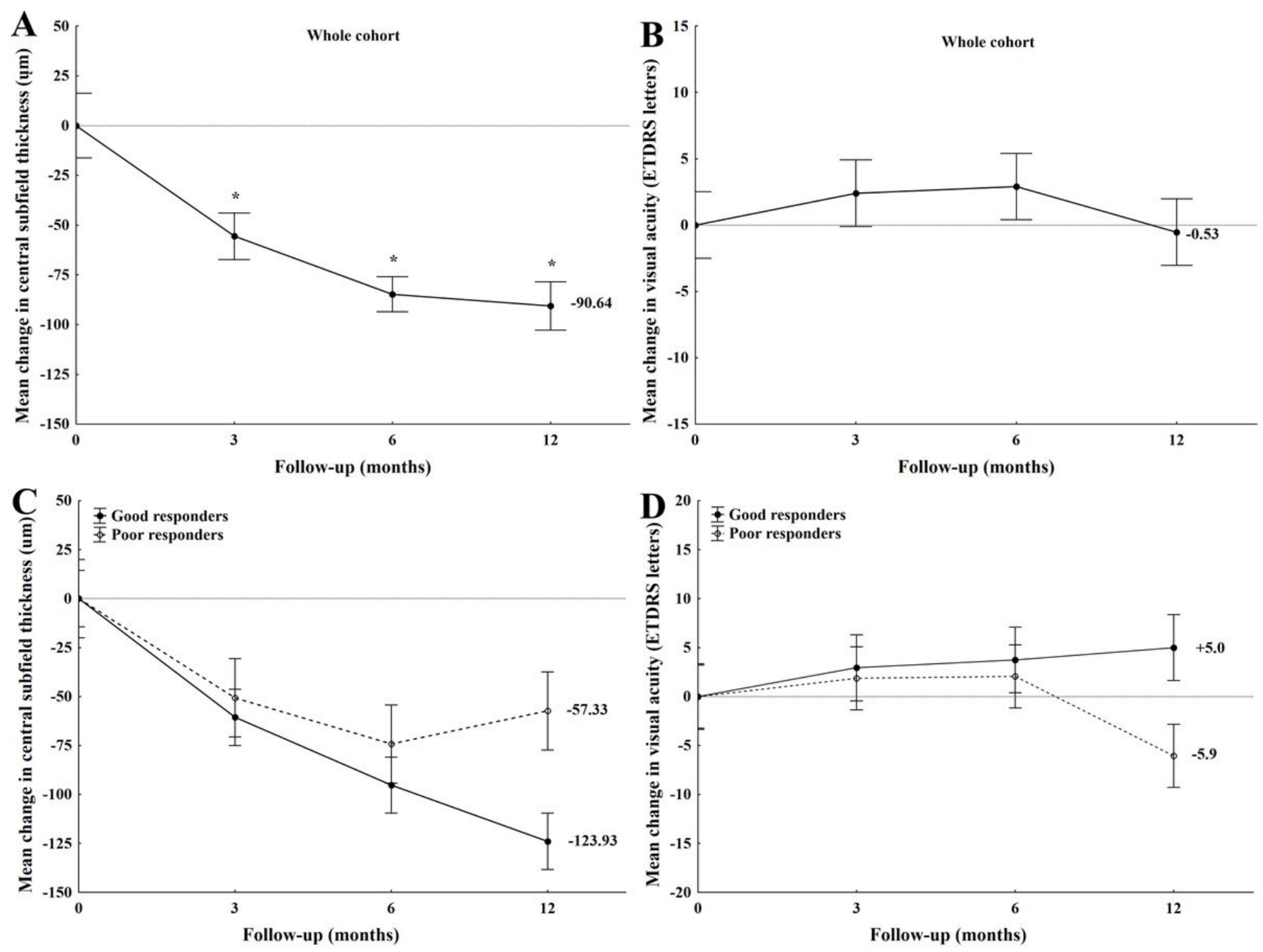

Figure 1

(A, B) Visual acuity and central subfield thickness (CSMT) changes in the whole cohort, (C, D) Visual acuity and central subfield thickness (CSMT) changes in the two study groups. 


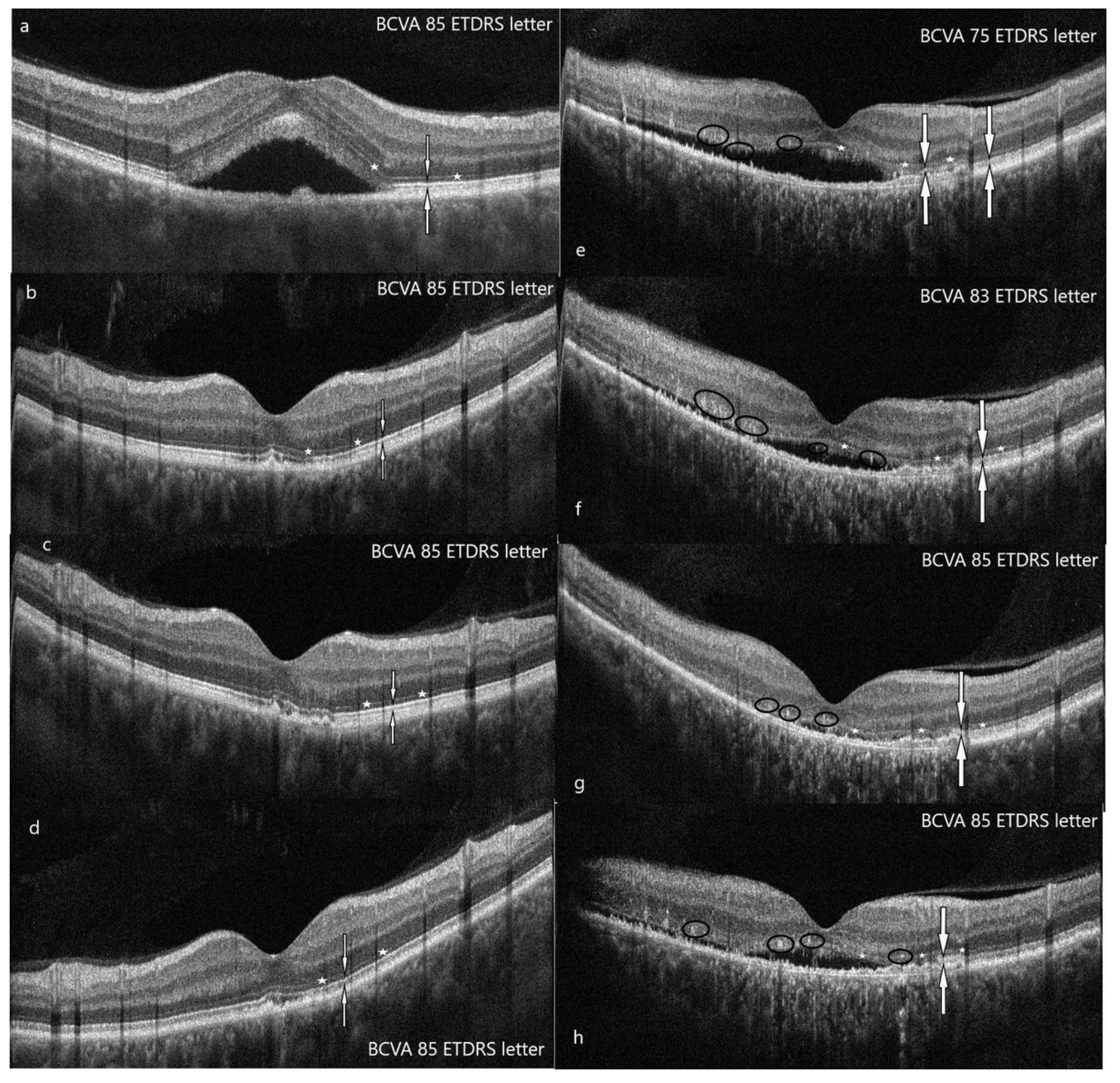

\section{Figure 2}

(a, b, c, d) Visual acuity change and OCT images of good responder to eplerenone at 0, 3, 6 and 12 months: at baseline SRF was seen which was absorbed after 3 months therapy and did not re-occure; ellipsoid is intact (line between white arrows), ELM is intact (little white stars). (e, f, g, h) Visual acuity change and OCT images of poor responder to eplerenone at $0,3,6$, and 12 months: ellipsoid zone is interrupted (line between white arrows), hyperreflective foci in the OS and ON layer, ELM is slightly damaged (little white stars). 